\title{
A New Propulsion System for Microswimmer Robot and Optimizing Geometrical Parameters Using PSO Algorithm
}

\author{
Hassan Sayyaadi ${ }^{1 *}$, Abolfazl Motekallem ${ }^{2}$ \\ ${ }^{1}$ Professor, Sharif University of Technology, Center of Excellence in Hydrodynamics and Dynamics of \\ Marine Vehicles, School of Mechanical Engineering; Sayyaadi@Sharif.Ir \\ ${ }^{2}$ M.Sc. Student, Sharif University of Technology, School of Mechanical Engineering; \\ Motekallem_Abolfazl@Mech.Sharif.Ir
}

\section{ARTICLE INFO}

Article History:

Received: 26 Dec. 2016

Accepted: 21 Sep. 2017

\section{Keywords:}

Biomimetic Microrobot

Swimming Robots

Interventional Therapy

Drug Delivery

Particle Swarm Optimization

\begin{abstract}
Mini and micro robots, which can swim in an underwater environment, have drawn widespread research interests because of their potential applications to the clinical drug delivery, biotechnology, manufacturing, mobile sensor networks, etc. In this paper, a prototype of microrobot based on the motion principle of living microorganisms such as E. Coli Bacteria is presented. The properties of this propulsive mechanism are estimated by modeling the dynamics of the swimming methods. For dynamic modeling and analysis of a tiny microrobot, which composed of a spherical head and four helix tail, the resistance force theory (RFT) is used to calculate thrust force, required torque, linear and angular velocities and then these physical and geometrical parameters are used to optimize the microrobot. In addition, a novel design method for determining the optimal geometrical parameters of dynamic system using the particle swarm optimization (PSO) reinforcement evolutionary algorithm is presented. Finally, the dynamical behavior of the optimized microrobot are simulated and the results are presented.
\end{abstract}

\section{Introduction}

Advances in micro / nano manufacturing technology have allowed scientists and engineers to take inspiration from locomotion strategies found in nature and design artificial swimmers and fluid transport systems at small scales. Swimming microrobots are mobile, untethered devices that have a typical size in the sub millimeter range i.e. from one millimeter down to one micrometer [1], and are able to move within a liquid medium [2]. The envisioned applications of swimming microrobots are mainly found in medicine, especially involving navigation in bodily fluids, such as blood and cerebrospinal fluid, for the execution of vary localized diagnosis or therapy tasks [3]. Swimming microrobots typically operated in the low Reynolds number regime, where inertia plays a minor role with respect to viscous damping. Swimming at these scales thus requires mechanisms that are substantially different from those employed at the macro scale [4]. Therefore designing a swimming microrobot is certainly a challenging task. A viable and effective way to cope with this issue is to exploit nature as a source of inspiration. Microorganisms are indeed able to effectively swim at the micro scale by adopting different techniques [1]. Bacteria are absolutely the most biological models in swimming microrobots. Tiny microrobots have been developed that swim by means of helical tails resembling bacterial flagella [56]. Inspired by the nature, several propulsion mechanisms have been proposed for swimming microrobot [7-11]. Whereas macro scale biomimetic robots rely on inertial forces for propulsion, miniaturization will make them ineffective [7]. In another design, an external magnetic field is used to rotate a small ferromagnetic screw within the liquid [11]. It has been demonstrated that this spiral machine can swim in liquids of various viscosities in a broad range of Reynolds numbers. However, speed limitation is the main deficiency of this machine.

Some existing approaches to mobile microrobot power and control in 3D such as chemically propelled designs include the microtubular jet microrobot [12], and the electroosmotic swimmer [13], Swimming microrobots include the colloidal magnetic swimmer [14], the magnetic thin film helical swimmer [15], the micro scale magnetic helix fabricated by glancing angle deposition [16], the micro helix microrobot with cargo carrying cage, fabricated by direct laser writing [17] and the micro helix microrobot with magnetic head, fabricated as thin film and rolled using residual 
stress [18], microrobots pulled in 3D using magnetic field gradients include the nickel microrobot capable of 5 DOF motion in 3D using the OctoMag system [19] and the MRI powered and imaged magnetic bead [20], Bio hybrid approaches include the artificially magnetotactic bacteria [21], the chemotactic steering of bacteria propelled microbeads [22] and the bacteria swarm manipulating micron scale bricks [23].

This research intends to investigate the potential of helix tail propulsion for designing a biomimetic swimmers. The dynamic model consists of a spherical body and four helical filament. The linear and angular swimming velocity of this model was theoretically predicted by using resistance force theory.

\section{Bionic Design of the Microrobot}

In order to design the microrobot for medical applications, the motion mode and the configuration of microorganisms have been studied. It is found that the microorganisms have a flexible tails and that the microorganisms have two swimming modes: one is the slender type organism's undulatory swimming mode such as the tadpoles swimming; the other is the spinning propulsive mode which is similar to the bacteria swimming with flagella. Therefore, we propose a novel noninvasive propulsion system inspired by bacteria. The microrobot is composed of an array of rigid tails and head where the driving motors are assembled in microrobot head.

To increase the thrust of a microrobot propelled by flagellar propulsion, we propose using multiple flagella in parallel as shown conceptually in Figure 1.

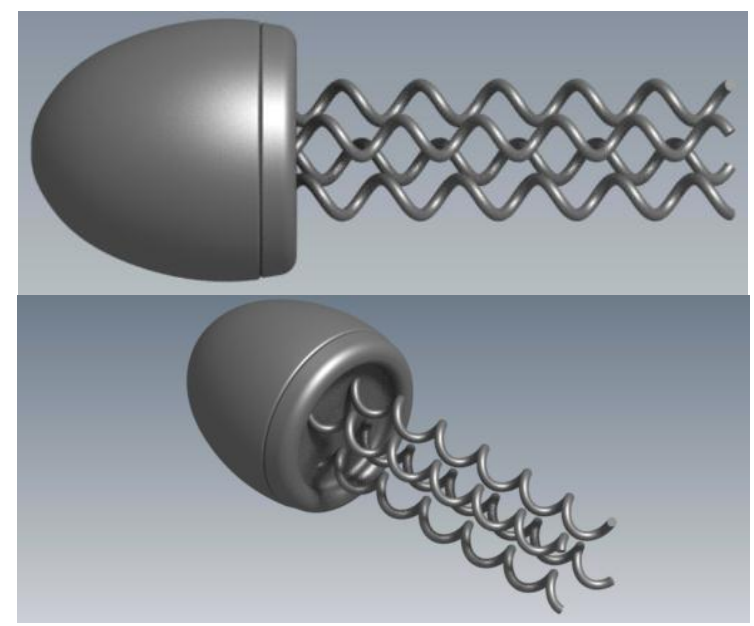

Figure 1. Conceptual Drawing Of The Interventional Swimming Microrobot

Multiple micro scale flagella are proposed to be made parallel to each other on a base. Designed microrobot is similar the biological analogue, which requires that each flagellum be rotated independently. The reasons for this are that rotating each flagellum independently is more difficult but the maneuver and kinematic parameters increased. The whole surface of the microrobot is biocompatible. When the flexible tails spin in liquid surroundings the thrust force will be generated due to the viscous force of the liquid applying to the tails. As we unitize the rotation direction and speed of the tails, the swimming direction will be adjusted easily [24-25].

\section{Dynamic Model of the Swimming Microrobot}

To develop an appropriate propulsion system, it is essential to build the dynamic model of the propulsion method. Based upon the application, the miniature medical microrobot is designed for and according to the maximal diameter of human's artery, the diameter of the microrobot must be less than $3 \mathrm{~mm}$. Since the viscosity of plasma is $0.01(N \cdot s) / \mathrm{m}^{2}$, we anticipate that the $R e$ of the microrobot would be much smaller than 1. The fluid flow of this regime is called Stokes flow. Main characteristics of Stokes flow are that the viscous forces are dominant over the inertial forces, and the propulsion depends on the interaction static effect between the microrobot and the fluid, and that the motion is time independent. So, utilize the previously studies on hydrodynamics of the microorganisms swimming in Stokes flow regime, the dynamic model of the propulsion has been developed. As shown in Figure 2, the rigid spinning tail of microrobot will be formed as a helical structure which is separated from many elements, and the forces between the elements and the fluid are called the resistance forces. Based on the Resistance Force Theory (RFT) given by Gray and Hancock [26-27], the normal and tangential components of drag force on every element of the slender body are proportional to the respective component of the local velocity with different proportionality constants, as the $R e$ is less than 1 .

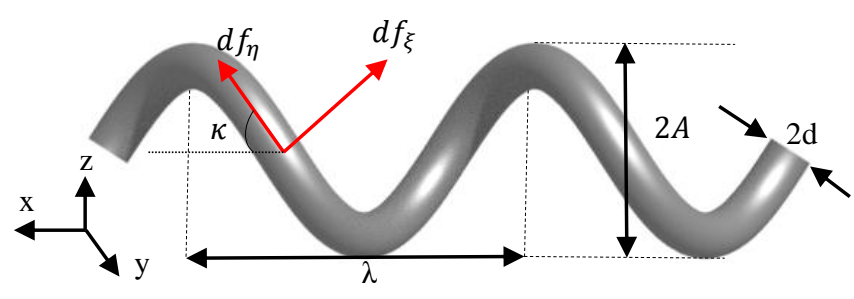

Fig . 2. The Simplified Model of the Helical Tail Of Microrobot

Resistive force theory states that the normal and tangential forces acting on a cylindrical element of length $d s$ are

$$
\begin{aligned}
& d f_{\xi}=-C_{\perp} v_{\xi} d s \\
& d f_{\eta}=-C_{\|} v_{\eta} d s
\end{aligned}
$$

Where $v_{\xi}$ and $v_{\eta}$ are respectively the normal and tangential velocities of the element and $C_{\perp}$ and $C_{\|}$are coefficients of resistance for a flagellum defined as [28]

$$
C_{\perp}=\frac{4 \pi \mu}{\ln \left(\frac{0 \cdot 18 \lambda}{\operatorname{dcos}(\kappa)}\right)+\frac{1}{2}}
$$




$$
C_{\|}=\frac{2 \pi \mu}{\ln \left(\frac{0 \cdot 18 \lambda}{d \cos (\kappa)}\right)}
$$

Here, $\lambda$ is the flagella helix wavelength, $\mu$ is the fluid's dynamic viscosity and $d$ is the flagellum wire radius as shown in Figure 2. Now, composing $\dot{x}$ and $A(\omega-\dot{\alpha})$ in normal and tangential directions, $v_{\xi}$ and $v_{\eta}$ can be written in terms of $\dot{x}$ and $A(\omega-\dot{\alpha})$ as

$$
\left[\begin{array}{l}
v_{\xi} \\
v_{\eta}
\end{array}\right]=\left[\begin{array}{cc}
\cos (\kappa) & -\sin (\kappa) \\
\sin (\kappa) & \cos (\kappa)
\end{array}\right]\left[\begin{array}{c}
A(\omega-\dot{\alpha}) \\
\dot{x}
\end{array}\right]
$$

$\omega$ is the angular velocity of the flagellum, $\dot{x}$ is the swimming speed of the microrobot, $\dot{\alpha}$ the angular velocity of a head, $A$ is the individual flagella amplitude and $\kappa$ is the pitch angle which is defined by

$$
\kappa=\tan ^{-1}\left(\frac{2 \pi A}{\lambda}\right)
$$

Substituting Eq. (5) into Eq. (1) and Eq. (2) the normal and tangential forces obtain as

$$
\begin{gathered}
{\left[\begin{array}{l}
d f_{\xi} \\
d f_{\eta}
\end{array}\right]=\left[\begin{array}{cc}
-C_{\perp} & 0 \\
0 & -C_{\|}
\end{array}\right]\left[\begin{array}{cc}
\cos (\kappa) & -\sin (\kappa) \\
\sin (\kappa) & \cos (\kappa)
\end{array}\right]} \\
{\left[\begin{array}{c}
A(\omega-\dot{\alpha}) \\
\dot{x}
\end{array}\right] d s}
\end{gathered}
$$

The length of $d s$ element in $x$ direction is

$$
d s^{2}=d x^{2}+d y^{2} \rightarrow d s=\frac{d x}{\cos (\kappa)}
$$

There would be an additional viscous torque acting on element $d s$ of the tail that results from the fluid reaction on the element of the tail. The $x$ component of this torque is [29]

$$
M_{S}=4 \pi \mu d^{2} \dot{\alpha} \cos (\kappa)
$$

The force and torque along the $x$ direction is derived as

$$
\left[\begin{array}{l}
d f_{x} \\
d M_{x}
\end{array}\right]=\left[\begin{array}{cc}
\cos (\kappa) & -\sin (\kappa) \\
\operatorname{Asin}(\kappa) & \operatorname{Acos}(\kappa)
\end{array}\right]\left[\begin{array}{l}
d f_{\eta} \\
d f_{\xi}
\end{array}\right]
$$

Substituting Eq. (7) into Eq. (10) the force and torque along the $x$ direction is derived as

$$
\begin{aligned}
& d f_{x}=-C_{\|}[\dot{x} \cos (\kappa)+A(\omega-\dot{\alpha}) \sin (\kappa)] d x \\
& -C_{\perp}[\dot{x} \sin (\kappa)-A(\omega-\dot{\alpha}) \cos (\kappa)] d x \tan (\kappa) \\
& d M_{x}=-A C_{\|}[\dot{x} \cos (\kappa)+A(\omega-\dot{\alpha}) \sin (\kappa)] d x \tan (\kappa) \\
& +A C_{\perp}[\dot{x} \sin (\kappa)-A(\omega-\dot{\alpha}) \cos (\kappa)]
\end{aligned}
$$

If the number of the helicon waves formed by the tail is $n$, by integration of Eq. (11), the thrust force of every flagellum can be written as

$$
\begin{aligned}
& F_{x}=\int_{0}^{n \lambda} d f_{x} \\
& F_{x}=-n \lambda C_{\|}[\dot{x} \cos (\kappa)+A(\omega-\dot{\alpha}) \sin (\kappa)] \\
& -n \lambda C_{\perp}[\dot{x} \sin (\kappa)-A(\omega-\dot{\alpha}) \cos (\kappa)] \tan (\kappa)
\end{aligned}
$$

By integrating of Eq. (12) the required torque of every flagellum can be written as

$$
\begin{aligned}
& M_{x}=\int_{0}^{n \lambda} d M_{x} \\
& M_{x}=-A n \lambda C_{\|}[\dot{x} \cos (\kappa) \\
& +A(\omega-\dot{\alpha}) \sin (\kappa)] \tan (\kappa) \\
& +A n \lambda C_{\perp}[\dot{x} \sin (\kappa)-A(\omega-\dot{\alpha}) \cos (\kappa)]
\end{aligned}
$$

The position of the flagella and free body diagram of the swimming microrobot are shown in Figure 3.

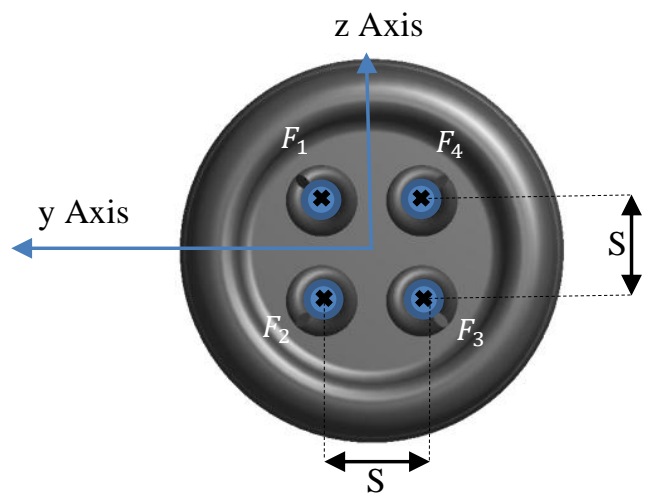

Figure 3. The Arrangement of Flagellum

The equations for conservation of linear and angular momentum in the $x$ direction can be written as

$$
\begin{aligned}
& \sum f_{\text {x.tail }}-f_{\text {Head }}=0 \\
& \sum M_{\text {x.tail }}-M_{\text {Head }}=0
\end{aligned}
$$

If the head of the microrobot is modeled as a sphere, $f_{\text {Head }}$ and $M_{\text {Head }}$ are calculated as

$$
\begin{aligned}
& f_{\text {Head }}=6 \pi \mu a \dot{x} \\
& M_{\text {Head }}=8 \pi \mu a^{3} \Omega
\end{aligned}
$$

Where $\Omega$ is an angular velocity and $a$ is the radius of the head. Therefore, the equilibrium equations can be written as 


$$
\begin{aligned}
& \sum_{i=1}^{4} F_{i . x}-6 \pi \mu a \dot{x}=0 \\
& \sum_{i=1}^{4}\left[M_{i . x}+M_{i . S}\right]-8 \pi \mu a^{3} \dot{\alpha}=0 \\
& \frac{S}{2}\left[F_{1 . x}+F_{4 . x}\right]-\frac{S}{2}\left[F_{2 . x}+F_{3 . x}\right]-8 \pi \mu a^{3} \dot{\beta}=0 \\
& \frac{S}{2}\left[F_{3 . x}+F_{4 . x}\right]-\frac{S}{2}\left[F_{1 . x}+F_{2 . x}\right]-8 \pi \mu a^{3} \dot{\gamma}=0
\end{aligned}
$$

As shown in Figure 4 the angular velocities about the $x, y$ and $z$ axes are specified as $\dot{\alpha}, \dot{\beta}$ and $\dot{\gamma}$, respectively.

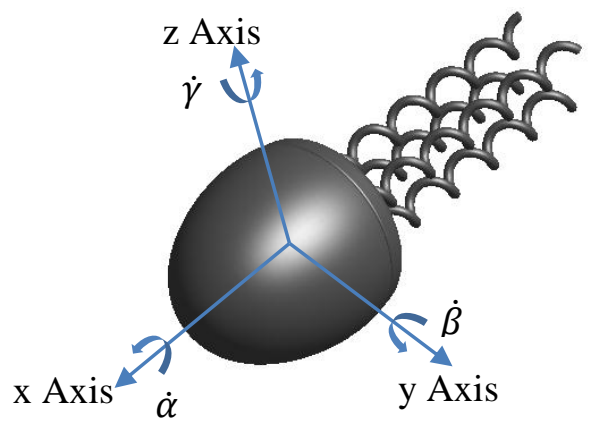

Figure 4. Angular Velocity In Body Coordinates

Substituting Eq. (13), Eq. (14) and Eq. (9) into Eq. (19) to Eq. (21) and using mathematical solver the propulsion velocity and angular velocities of microrobot in body coordinates are obtained as

$$
\begin{aligned}
& \dot{x}=\frac{f(A \cdot n \cdot d \cdot \lambda)}{g(A \cdot n \cdot d \cdot \lambda)} \sum_{i=1}^{4} \omega_{i} \\
& \dot{\alpha}=\frac{h(A \cdot n \cdot d \cdot \lambda)}{g(A \cdot n \cdot d \cdot \lambda)} \sum_{i=1}^{4} \omega_{i} \\
& \dot{\beta}=r(\text { A.n.d. } \lambda)\left[\omega_{1}+\omega_{4}-\omega_{2}-\omega_{3}\right] \\
& \dot{\gamma}=r(A . n . d . \lambda)\left[\omega_{3}+\omega_{4}-\omega_{1}-\omega_{2}\right]
\end{aligned}
$$

The functions defined in Eq. (23) to Eq. (26) are derived as

$$
\begin{aligned}
& f(A . n . d . \lambda)=A \sin (\kappa)\left[\lambda n \pi d^{2} \cos (\kappa)\right. \\
& \left.-2 \pi \mu a^{3}\right]\left(C_{\perp}-C_{\|}\right) \\
& g(A . n . d \cdot \lambda)=4\left[A^{2} \lambda n C_{\perp} C_{\|} \sec ^{2}(\kappa)\right. \\
& +\pi \mu\left(C_{\|} \cos ^{2}(\kappa)+C_{\perp} \sin ^{2}(\kappa)\right)\left(\lambda d^{2} n-\right. \\
& \left.\left.2 a^{3} \sec (\kappa)\right)\right] \\
& h(\text { A.n.d. } \lambda)=A^{2} \lambda n C_{\perp} C_{\|} \sec ^{2}(\kappa)
\end{aligned}
$$

$$
r(A . n . d . \lambda)=\frac{\operatorname{SAn\lambda }\left(C_{\perp}-C_{\|}\right) \sin (\kappa)}{16 \pi \mu a^{3}}
$$

The Eq. (23) to Eq. (26) have been developed in body coordinates. By defining $(X, Y, Z)$ as inertia coordinates therefore transition from body coordinates to inertia coordinates performed using Eq. (31).

$$
\left[\begin{array}{l}
I \\
J \\
K
\end{array}\right]=\left(R_{\alpha}\right)^{T}\left(R_{\beta}\right)^{T}\left(R_{\gamma}\right)^{T}\left[\begin{array}{l}
i \\
j \\
k
\end{array}\right]=R_{\text {Trans }}\left[\begin{array}{l}
i \\
j \\
k
\end{array}\right]
$$

The $R_{\text {Trans }}$ is defined as

$$
R_{\text {Trans }}=\left[\begin{array}{ccc}
c \gamma c \beta & -s \gamma c \alpha+c \gamma s \beta s \alpha & s \gamma s \alpha+c \gamma s \beta c \alpha \\
s \gamma c \beta & c \gamma c \alpha+s \alpha s \beta s \gamma & -c \gamma s \alpha+s \gamma s \beta c \alpha \\
-s \beta & c \beta s \alpha & c \beta c \alpha
\end{array}\right]
$$

Where $s=\sin$ and $c=\cos$.

The linear and angular velocities of microrobot in inertia coordinates are obtained as below

$$
\begin{aligned}
& \dot{X}=\dot{x} \cos (\gamma) \cos (\beta) \\
& \dot{Y}=\dot{x} \cos (\gamma) \sin (\beta) \\
& \dot{Z}=-\dot{x} \sin (\gamma) \\
& \dot{\Theta}=[\cos (\gamma) \cos (\beta)] \dot{\alpha} \\
& -[\sin (\gamma) \cos (\alpha)+\cos (\gamma) \sin (\beta) \sin (\alpha)] \dot{\beta} \\
& +[\cos (\gamma) \sin (\beta) \cos (\alpha)-\sin (\gamma) \sin (\alpha)] \dot{\gamma} \\
& \dot{\Psi}=[\sin (\gamma) \cos (\beta)] \dot{\alpha} \\
& +[\cos (\gamma) \cos (\alpha)-\sin (\alpha) \sin (\beta) \sin (\gamma)] \dot{\beta} \\
& +[\sin (\gamma) \sin (\beta) \cos (\alpha)-\cos (\gamma) \sin (\alpha)] \dot{\gamma} \\
& \dot{\Phi}=-[\sin (\beta)] \dot{\alpha}-[\cos (\beta) \sin (\alpha)] \dot{\beta} \\
& \quad+[\cos (\beta) \cos (\alpha)] \dot{\gamma}
\end{aligned}
$$

It should be noted that any change in angular velocity $\left(\omega_{i}\right)$ of each flagellum increases or decreases the linear and angular velocities of microrobot. For example, when the angular velocities of all flagellum are equal, the designed microrobot will move on straight line. In this case, each of the flagellum generated the same force, therefore any torque about the coordinates axes will occur. This causes that the microrobot without any precession, move in straight line which it is visible in equilibrium equations (19) to (21).

\section{Dynamic Performance Analysis}

The dynamic properties of the robot varying with the characteristic geometrical parameters of the flagellum, the force and required torque are analyzed. In the 
theoretical analysis, the viscosity of fluid is set to $0.01(\mathrm{~N} \cdot \mathrm{s}) / \mathrm{m}^{2}$, which approaches to the viscosity of plasma. Other parameters are chosen according the dimensions of living microorganisms.

In Figure 5, the thrust force and the required torque are plotted as a function of the helical amplitude $A$ at the constants $\mu=0.01 \frac{N \cdot s}{m^{2}}, d=0.0115 \mu m, n=10, \lambda=$ $0.38 \mu \mathrm{m}, \omega=100 \frac{\mathrm{rad}}{\mathrm{s}}$.

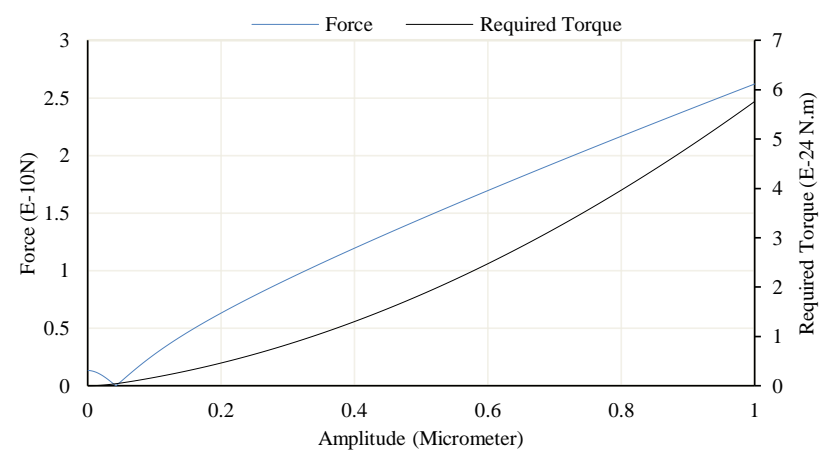

Figure 5. The Thrust Force and the Required Torque of Motor As a Function of Amplitude

It shows that as the helical amplitude increases, the thrust force and required torque of the motor increase. When the helical amplitude is increasing from zero to certain value, the thrust force dramatically increase, while the increment of required torque is small. When the helical amplitude increases over the range, the increasing rates of the torque is much higher than thrust force.

In Figure 6, the thrust force and the required torque are plotted as a function of the helical wavelength at the constants $\mu=0.01 \frac{\mathrm{N} \cdot \mathrm{s}}{\mathrm{m}^{2}}, d=0.0115 \mu \mathrm{m}, n=10, A=$ $0.33 \mu \mathrm{m}, \omega=100 \frac{\mathrm{rad}}{\mathrm{s}}$.

This plot shows that as $\lambda$ increases, the thrust force and required torque dramatically increase. Increasing rates of the thrust force and required torque is equal. When the helical wavelength increases over the range, the thrust force leads to required torque.

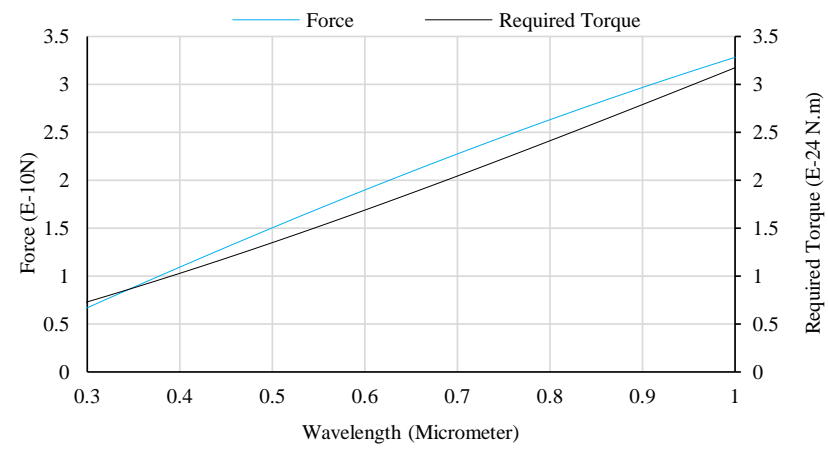

Figure 6. The Thrust Force and the Required Torque of Motor As a Function of Wavelength

In Figure 7, the thrust force and the required torque are plotted as a function of the flagellum radius $d$ at the constants $\mu=0.01 \frac{N \cdot s}{m^{2}}, A=0.33 \mu m, n=10, \lambda=$ $0.38 \mu \mathrm{m}, \omega=100 \frac{\mathrm{rad}}{\mathrm{s}}$.

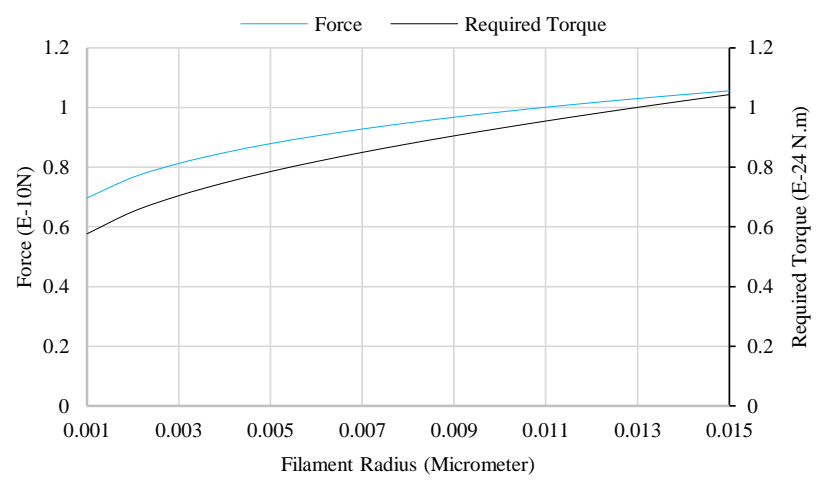

Figure 7. The Thrust Force and the Required Torque of Motor As a Function of Flagellum Radius

This plot shows that as flagellum radius increases, the increasing rate of the thrust force more than the required torque. Further flagellum radius increases, the increasing rate of the required torque and thrust force are approximately constant and leads to certain value. In Figure 8, the thrust force and the required torque are plotted as a function of the viscosity of fluid at the constants $A=0.33 \mu m, d=0.0115 \mu m, n=10, \lambda=$ $0.38 \mu \mathrm{m}, \omega=100 \frac{\mathrm{rad}}{\mathrm{s}}$.

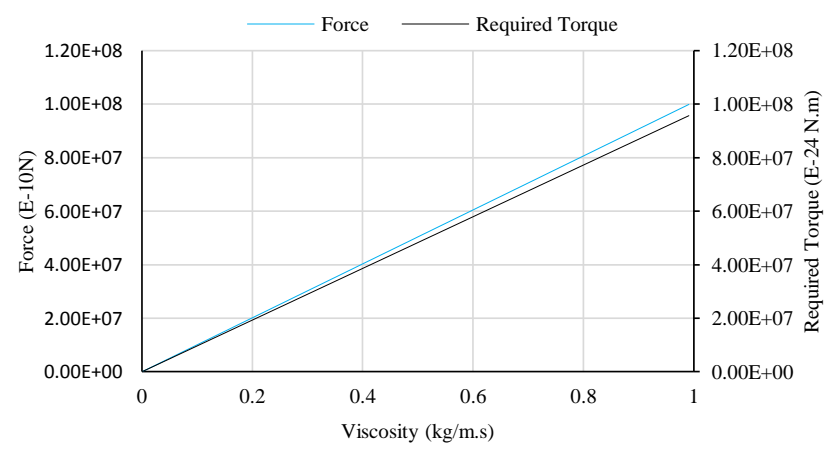

Figure 8. The Thrust Force and the Required Torque of Motor as a Function of Viscosity

This plot shows that as the viscosity of fluid increases, the thrust force and the required torque increase while the graphs slop is constant. The increasing tendencies of the thrust force and the required torque are coincident.

In Figure 9, the thrust force and the required torque are plotted as a function of angular velocity of the motor at the constants $\quad \mu=0.01 \frac{N \cdot S}{\mathrm{~m}^{2}}, A=0.33 \mu \mathrm{m}, \quad d=$ $0.0 .0115 \mu \mathrm{m}, n=10, \lambda=0.38 \mu \mathrm{m}$.

This plot shows that as angular velocity increases, the thrust force and the required torque are increase. The increasing rates of the thrust force and the torque are approximately constant. 


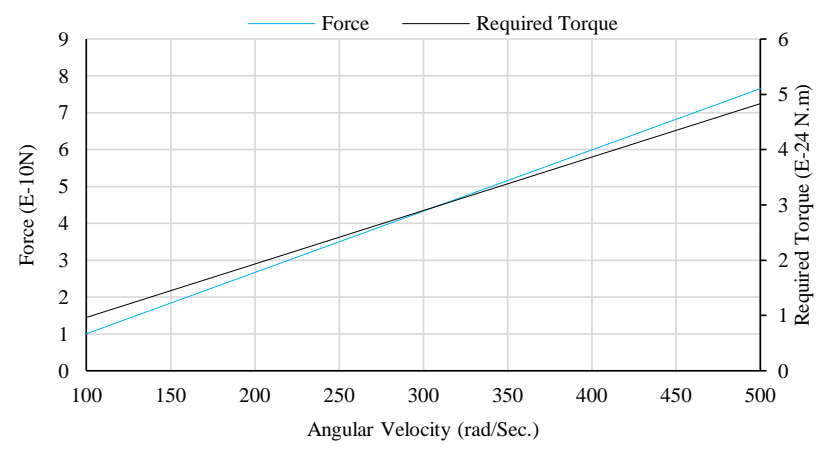

Figure 9. The Thrust Force and the Required Torque of Motor As a Function of Angular Velocity

In Figure 10, the thrust force and the required torque are plotted as a function of wave number $n$ at the constants $\mu=0.01 \frac{N \cdot s}{m^{2}}, d=0.0115 \mu m, A=$ $0.33 \mu \mathrm{m}, \lambda=0.38 \mu \mathrm{m}, \omega=100 \frac{\mathrm{rad}}{\mathrm{s}}$.

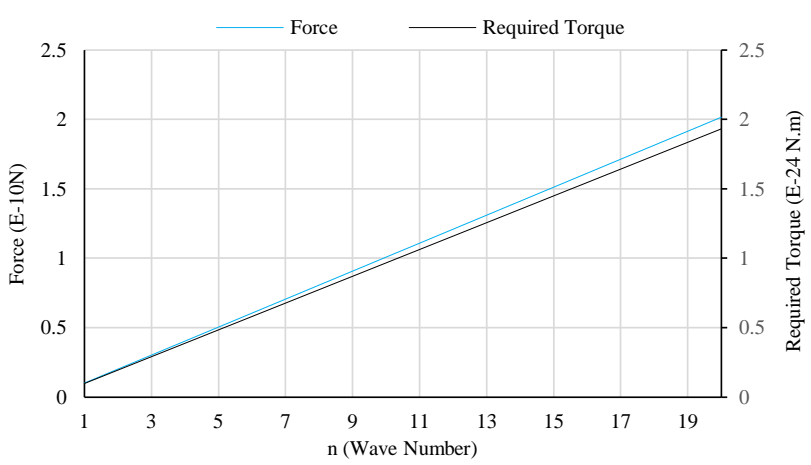

Figure 10. The Thrust Force and the Required Torque of Motor As a Function of Wave Number

This plot shows that as wave number of the tail increase, the thrust force and the required torque increase. The increasing rates of the thrust force and the torque are approximately constant.

\section{Particle Swarm Optimization (PSO)}

Kennedy and Eberhart [30] originally proposed the PSO algorithm for optimization. PSO is a population based search algorithm based on the simulation of the social behavior of birds within a flock. Although, this algorithm was originally adopted for balancing weights in neural networks [31], it soon became a very popular global optimizer, mainly in problems in which the decision variables are real numbers [32-33]. In PSO, particles fly throughout a hyper dimensional search space. Changes to the position of the particles within the search space are based on the social psychological tendency of individuals to emulate the success of other individuals. The positions of these particles are changed according to their own experience and that of their neighbors. Let $\overrightarrow{x_{l}}(t)$ denote the position of a particle. The position of $\overrightarrow{x_{l}}(t)$ is changed by adding a velocity $\overrightarrow{v_{l}}(t)$ to it, i.e.:

$$
\overrightarrow{x_{l}}(t+1)=\overrightarrow{x_{l}}(t)+\overrightarrow{v_{l}}(t+1)
$$

The velocity vector reflects the socially exchanged information and, in general, is defined in the following way:

$$
\begin{aligned}
& \overrightarrow{v_{l}}(t+1)=W \overrightarrow{v_{l}}(t)+C_{1} r_{1}\left(\vec{x}_{\text {peest }_{i}}-\overrightarrow{x_{l}}(t)\right) \\
& +C_{2} r_{2}\left(\vec{x}_{\text {gbest }_{i}}-\overrightarrow{x_{l}}(t)\right)
\end{aligned}
$$

Where $C_{1}$ is the cognitive learning factor and represents the attraction that a particle has toward its own success. $C_{2}$ is the social learning factor and represents the attraction that a particle has toward the success of the entire swarm. $W$ is the inertia weight which is employed to control the impact of the previous history of velocities on the current velocity of a given particle. $\vec{x}_{\text {pbest }_{i}}$ is the personal best position of the particle $i$. $\vec{x}_{\text {gbest }_{i}}$ is the position of the best particle of the entire swarm. $r_{1}, r_{2} \in[0,1]$ are random values.

In addition, throughout the paper a uniform probability distribution is assumed for all random parameters. The parameter $W$ regulates the tradeoff between the global and local exploration abilities of the swarm. A large inertia weight facilitates the global exploration, while a small one tends to facilitate the local exploration. A suitable value for the inertia weight balances between global and local exploration abilities. Experimental results indicate that the linearly decreasing inertia weight over the iterations improve the performance of PSO [34]. Furthermore, with a large value of $C_{1}$ and a small value of $C_{2}$, particles are allowed to move around their personal best position $\left(\vec{x}_{\text {pbest }}\right)$. With a small value of $C_{1}$ and a large value of $C_{2}$, particles converge to the best particle of the entire swarm $\left(\vec{x}_{\text {gbest }_{i}}\right)$. From the results, it was observed that best solutions were determined when $C_{1}$ is linearly decreased and $C_{2}$ is linearly increased over the iterations [35]. In this paper the inertia weight in first step time is assumption one and in other time step is defined as

$$
W(t+1)=W(t) \times w d a m p
$$

\section{Optimization}

Optimization problem is either single or multi objective depending on the number of their objective function. In this paper single objective optimization system consists of only one objective function prefers to increase the efficiency of propulsion microrobot. The objective function to be selected to required torque reduced and thrust force increase. Objective function is defined as below

$$
\Gamma=\Lambda M_{\text {Required }}-\Upsilon F_{\text {Thrust }}
$$

In Eq. (42) $\Lambda$ and $\Upsilon$ are two constant that $\Lambda$ and $\Upsilon \in$ [0 1]. The used parameters in PSO algorithm is shown in Table 1. The geometrical parameters of microrobot 
propulsion system is optimized consist of $n, A, \lambda$ and $d$.

Table 1. PSO Parameters

\begin{tabular}{cc}
\hline Parameters & Value \\
\hline$C_{1}$ & 2 \\
$C_{2}$ & 2 \\
$W$ & 1 \\
wdamp & 0.99 \\
\hline
\end{tabular}

Optimization results are shown in Table 2 and Table 3.

Table 2. Optimization Results

\begin{tabular}{cccccc}
\hline No. & $\Lambda$ & $\Upsilon$ & $\begin{array}{c}\text { Force } \\
(\text { E10-18) }\end{array}$ & $\begin{array}{c}\text { Moment } \\
(\mathrm{E} 10-24)\end{array}$ \\
\hline 1 & 0.05 & 0.95 & $9.29 \mathrm{E}-06$ & $2.26 \mathrm{E}-05$ \\
2 & 0.1 & 0.9 & $9.29 \mathrm{E}-06$ & $2.26 \mathrm{E}-05$ \\
3 & 0.15 & 0.85 & $8.53 \mathrm{E}-06$ & $1.79 \mathrm{E}-05$ \\
4 & 0.2 & 0.8 & $7.19 \mathrm{E}-06$ & $1.15 \mathrm{E}-05$ \\
5 & 0.25 & 0.75 & $6.19 \mathrm{E}-06$ & $8.01 \mathrm{E}-06$ \\
6 & 0.3 & 0.7 & $5.38 \mathrm{E}-06$ & $5.84 \mathrm{E}-06$ \\
7 & 0.35 & 0.65 & $4.67 \mathrm{E}-06$ & $4.37 \mathrm{E}-06$ \\
8 & 0.4 & 0.6 & $4.03 \mathrm{E}-06$ & $3.30 \mathrm{E}-06$ \\
9 & 0.45 & 0.55 & $3.44 \mathrm{E}-06$ & $2.49 \mathrm{E}-06$ \\
10 & 0.5 & 0.5 & $2.86 \mathrm{E}-06$ & $1.85 \mathrm{E}-06$ \\
11 & 0.55 & 0.45 & $2.03 \mathrm{E}-06$ & $1.11 \mathrm{E}-06$ \\
12 & 0.6 & 0.4 & $1.35 \mathrm{E}-06$ & $5.99 \mathrm{E}-07$ \\
13 & 0.65 & 0.35 & $8.80 \mathrm{E}-07$ & $3.16 \mathrm{E}-07$ \\
14 & 0.7 & 0.3 & $5.57 \mathrm{E}-07$ & $1.59 \mathrm{E}-07$ \\
15 & 0.75 & 0.25 & $3.37 \mathrm{E}-07$ & $7.49 \mathrm{E}-08$ \\
16 & 0.8 & 0.2 & $2.24 \mathrm{E}-07$ & $4.10 \mathrm{E}-08$ \\
17 & 0.85 & 0.15 & $1.68 \mathrm{E}-07$ & $2.85 \mathrm{E}-08$ \\
18 & 0.9 & 0.1 & $9.35 \mathrm{E}-09$ & $1.64 \mathrm{E}-09$ \\
19 & 0.95 & 0.05 & $5.87 \mathrm{E}-09$ & $1.37 \mathrm{E}-09$ \\
\hline
\end{tabular}

Table 3. Optimized Geometrical Parameters

\begin{tabular}{ccccc}
\hline No. & $\lambda(\mu m)$ & $\mathrm{A}(\mu \mathrm{m})$ & $\begin{array}{c}\text { Radius } \\
(\mu \mathrm{m})\end{array}$ & $\mathrm{n}$ \\
\hline 1 & 1.000 & 0.500 & 0.015 & 15.000 \\
2 & 1.000 & 0.500 & 0.015 & 15.000 \\
3 & 1.000 & 0.456 & 0.015 & 15.000 \\
4 & 1.000 & 0.381 & 0.015 & 15.000 \\
5 & 1.000 & 0.328 & 0.015 & 15.000 \\
6 & 1.000 & 0.286 & 0.015 & 15.000 \\
7 & 1.000 & 0.252 & 0.015 & 15.000 \\
8 & 1.000 & 0.222 & 0.015 & 15.000 \\
9 & 1.000 & 0.195 & 0.015 & 15.000 \\
10 & 1.000 & 0.170 & 0.015 & 15.000 \\
11 & 0.868 & 0.141 & 0.013 & 15.000 \\
12 & 0.707 & 0.115 & 0.010 & 15.000 \\
13 & 0.571 & 0.092 & 0.008 & 15.000 \\
14 & 0.454 & 0.073 & 0.006 & 15.000 \\
15 & 0.353 & 0.057 & 0.005 & 15.000 \\
16 & 0.289 & 0.050 & 0.002 & 15.000 \\
17 & 0.244 & 0.050 & 0.001 & 15.000 \\
18 & 0.183 & 0.050 & 0.001 & 1.115 \\
19 & 0.102 & 0.050 & 0.001 & 1.314 \\
\hline
\end{tabular}

Although all values of Table 3 are reasonable solutions but as can see the initial rows of Table 2 consist of great required torque, are not selected because the supply energy is dominated. The end rows consist of small thrust. Therefore No. 12 is optimal case and optimized geometrical parameters are shown in Table 3.

Table 3. Optimal Geometrical Parameters

\begin{tabular}{cccc}
\hline $\begin{array}{c}\text { Wavelength } \\
(\mu \mathrm{m})\end{array}$ & $\begin{array}{c}\text { Amplitude } \\
(\mu \mathrm{m})\end{array}$ & $\begin{array}{c}\text { Radius } \\
(\mu \mathrm{m})\end{array}$ & $\begin{array}{c}\text { Wave Number } \\
(\mu \mathrm{m})\end{array}$ \\
\hline 0.708 & 0.115 & 0.0108 & 15 \\
\hline
\end{tabular}

Figure 11 shows the objective function values $(\Gamma)$ of best global particle versus number of function evolution (NFE) for No. 12. As can be seen in Figure 11 by increasing NFE the objective function values of best global particle leads to constant value $(-2 \cdot 2 \times$ $\left.10^{-10}\right)$.

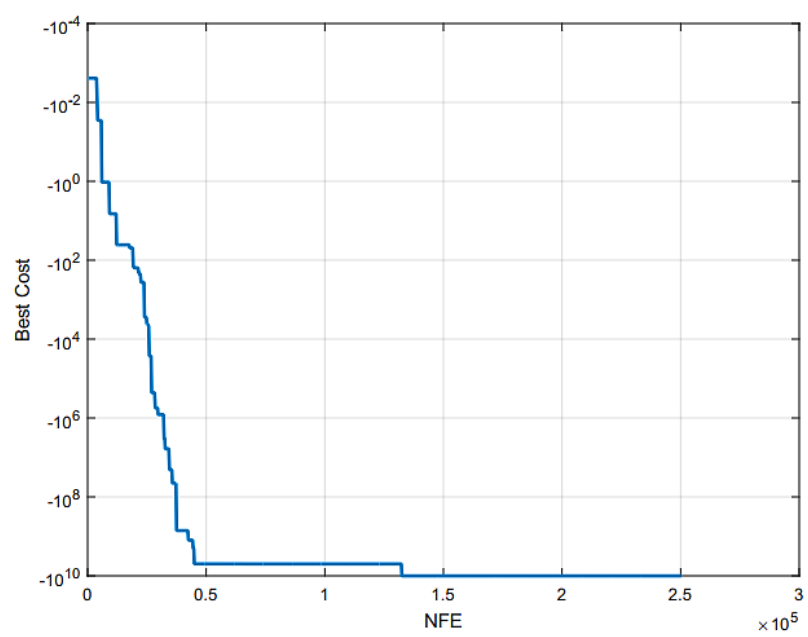

Figure 11. Best Cost Vs. Number of Function Evolution

\section{Dynamic Simulation Results for Optimized Microrobot System}

Simulations have been conducted using MATLAB software. The flagellum as a circular cylinder is characterized by its geometrical and material properties. The geometrical parameters of flagellum and microrobot values used in the simulation obtained from particle swarm optimization algorithm calculated in last section and living microorganism. The expressed Parameters are $A=0.500 \mu \mathrm{m}, \lambda=0.202 \mu \mathrm{m}, d=$ $0.060 \mu \mathrm{m}, n=15, \mu=0.010 \frac{\mathrm{N} \cdot \mathrm{S}}{\mathrm{m}^{2}}, a=1.800 \mu \mathrm{m}$ and $S=1.000 \mu m$.

When the angular velocity of all flagellums are equal and change linearly in time $\left(\omega_{i}=t\right)$, the designed microrobot will move in straight line (Figure 12). In this case, each flagellum generates the same force and therefore any torque about coordinates axes occur. This causes that the microrobot without any slope move in straight line. Figure 13 and Figure 14 show the linear velocity and angular velocity of the microrobot. 


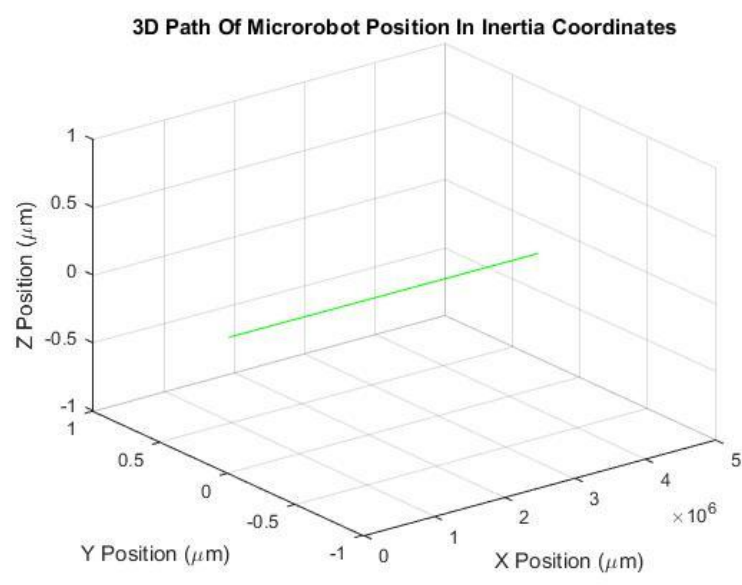

Figure 12. 3D Path of Microrobot
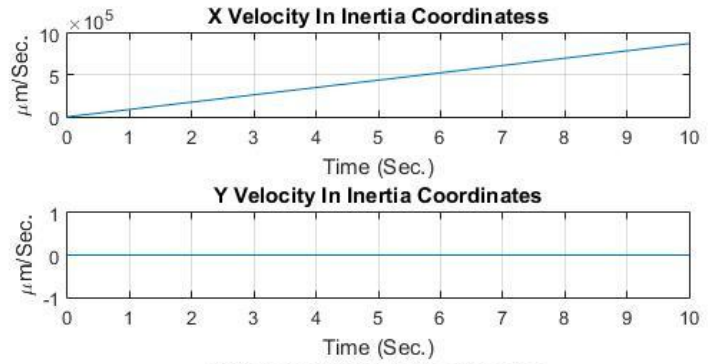

Z Velocity In Inertia Coordinates

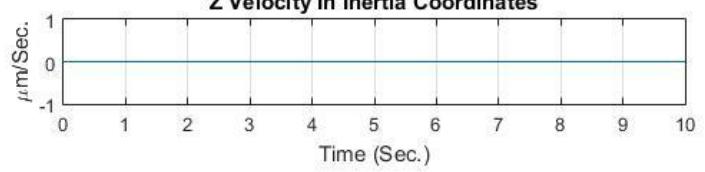

Figure 13. Linear Velocity In Inertia Coordinates
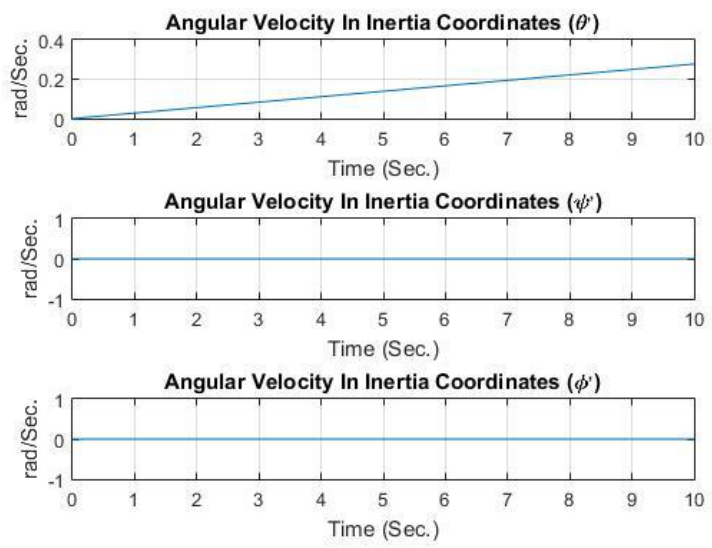

Figure 14. Angular Velocity In Inertia Coordinates

When the angular velocities of lateral flagellums are same and other meddle flagellum rotate in opposite direction $\left(\omega_{1}=\omega_{2}=\omega_{3} \neq \omega_{4}\right)$ then produce two equal torque about $\mathrm{Y}$ and $\mathrm{Z}$ axes with opposite signs. So the microrobot due to the torque about $\mathrm{X}$ axis fluctuate in $X-Y$ plane while the microrobot move along $X$ direction (Figure 15). Figure 16 and Figure 17 show the linear and angular velocity of microrobot.

$$
M_{Y}=-M_{Z}
$$

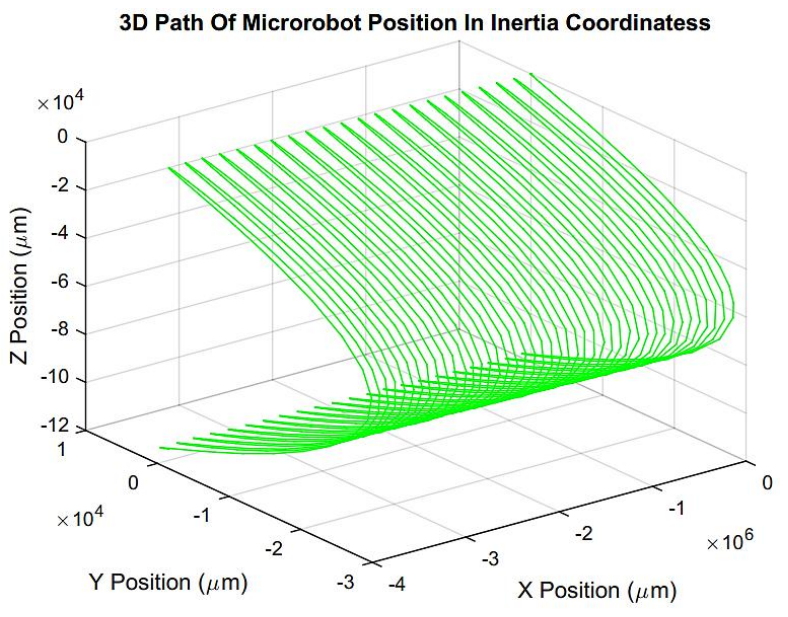

Figure 15. 3D Path of Microrobot
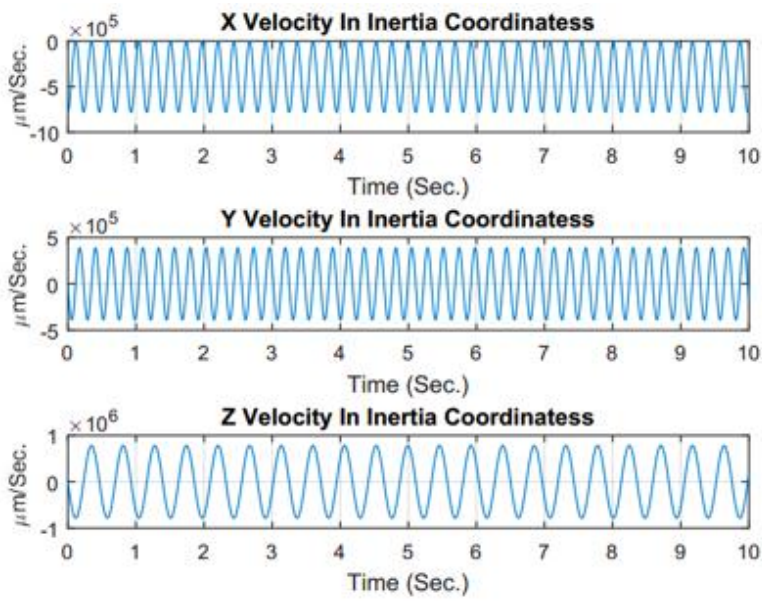

Figure 16. Linear Velocity In Inertia Coordinates
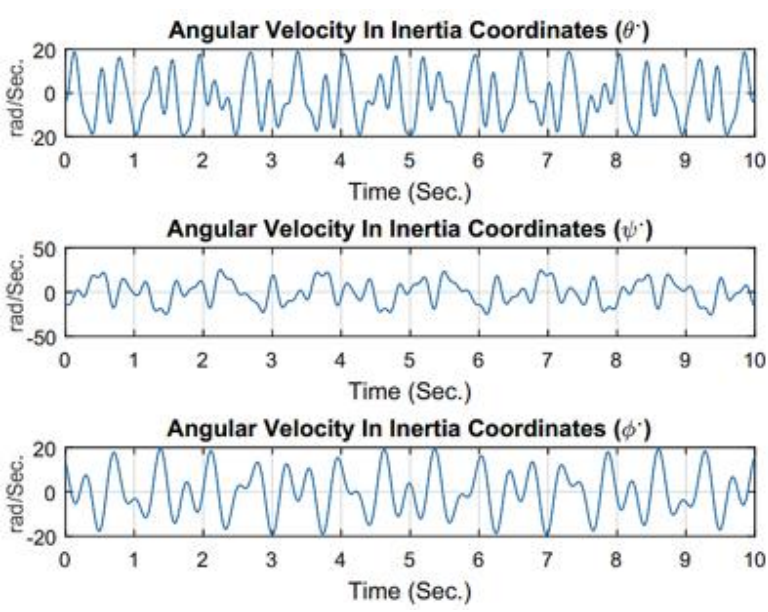

Figure 17. Angular Velocity In Inertia Coordinates

When the angular velocities of flagellums are as follows

$$
\omega_{1}=\omega_{2}=-\omega_{3}=-\omega_{4}
$$

Then the torque produced about coordinates axes and the linear velocities of microrobot are zero (Figure 19). Also the microrobot will not have any specific movement (Figure 18). It should be noted that in this situation the microrobot can rotate about $\mathrm{Z}$ axes (Figure 
20) which is a key factor in design of microrobot maneuvering.

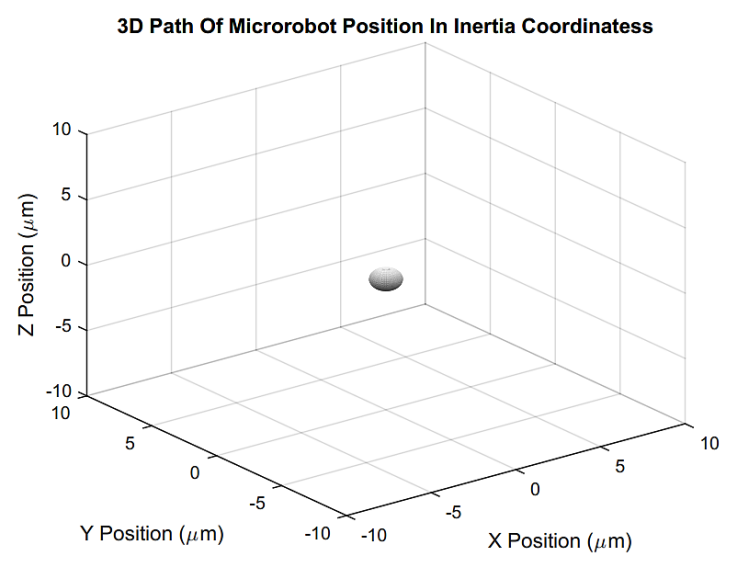

Figure 18. 3D Path of Microrobot

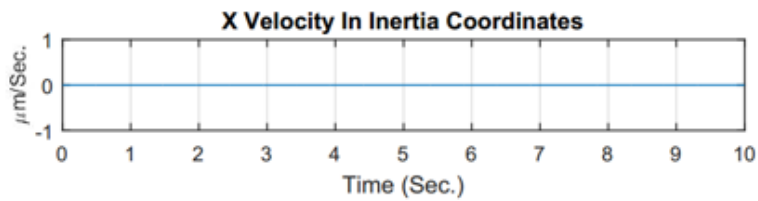

Y Velocity In Inertia Coordinates
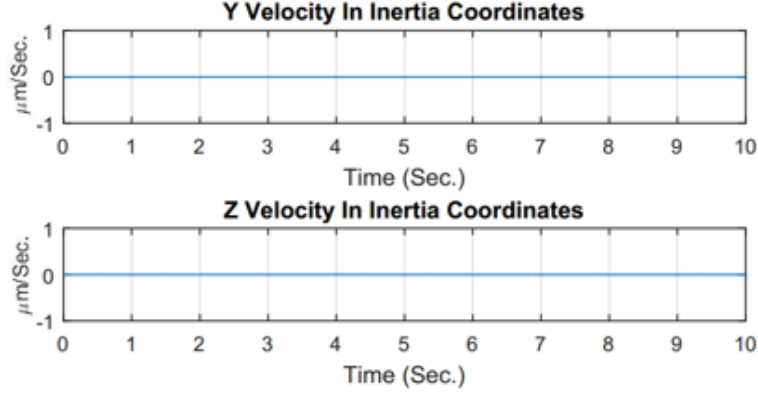

Figure 19. Linear Velocity In Inertia Coordinates
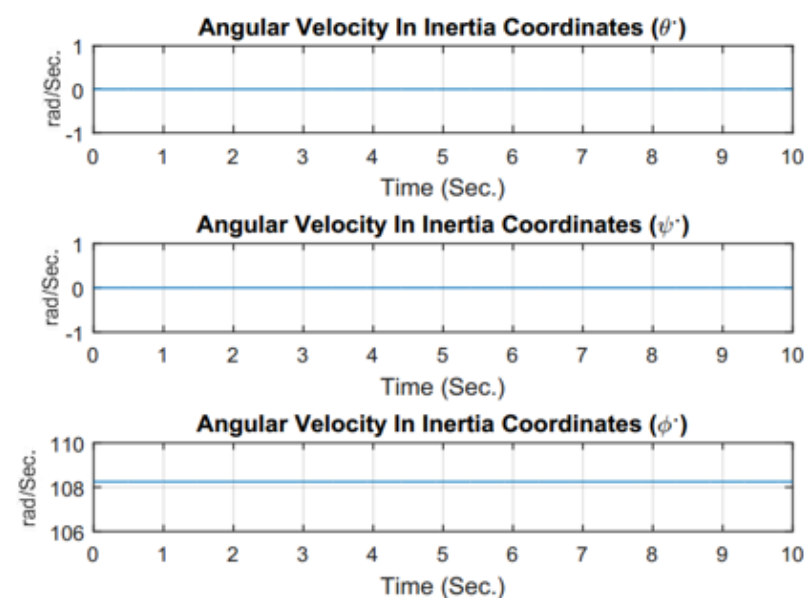

Figure 20. Angular Velocity In Inertia Coordinates

As illustrated in Fig. 21 when the angular velocities of flagellum are different, the microrobot is able to perform any three dimensional movement and maneuver in environment.

For example, when the angular velocities of flagellums are function of time and different with together, therefore the microrobot move on complex path. Figure 22 and Figure 23 show the linear and angular velocities of microrobot.

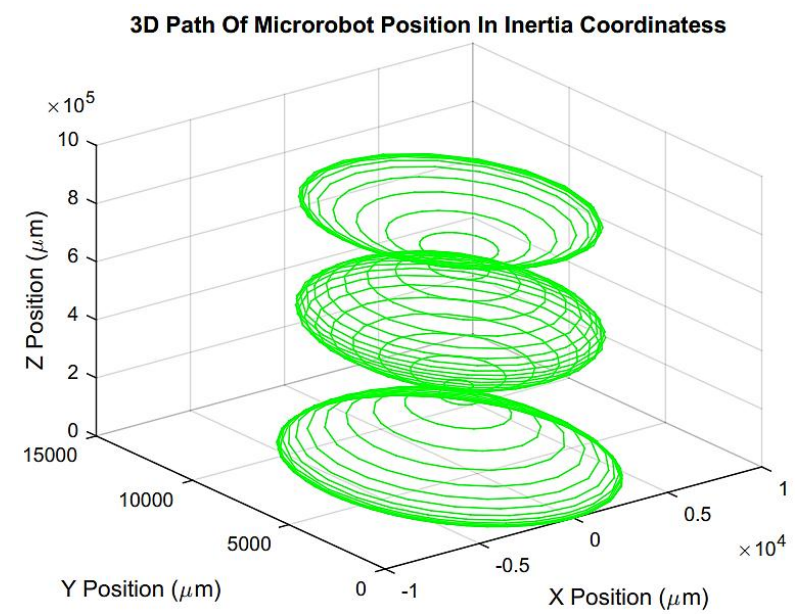

Figure 21. 3D Path of Microrobot
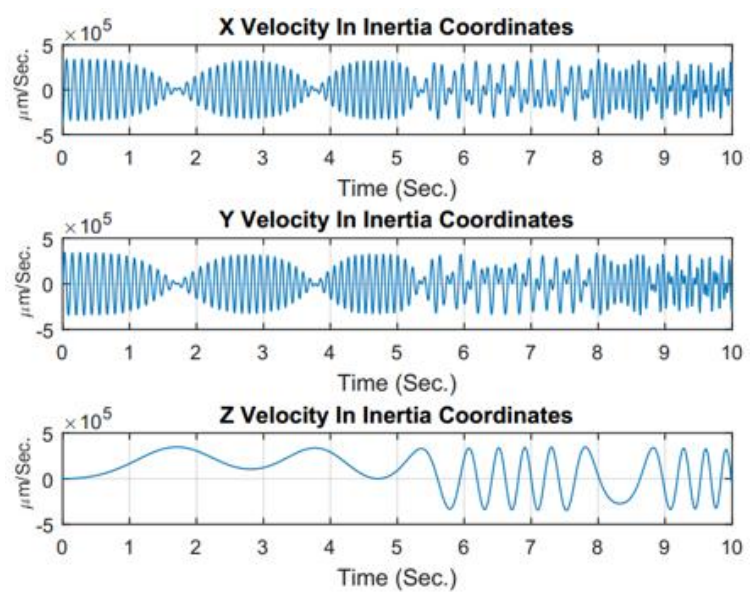

Figure 22. Linear Velocity In Inertia Coordinates
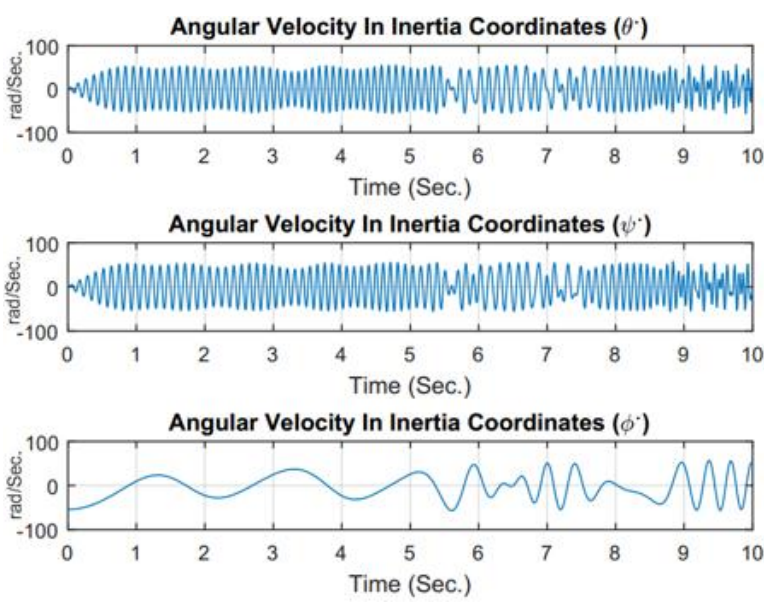

Figure 23 Angular Velocity In Inertia Coordinates

\section{Concluding Remarks}

Miniature, safe and energy efficient propulsion systems hold the key to mature this technology. In this paper, a prototype of microrobot based on the motion principle of living microorganisms is presented. Based on the RFT, the dynamic model of the swimming method has been made so as to analyze the thrust force achieved by the spinning flexible helical tails and the needed torque of the driving motor theoretically. The theoretical analysis indicates the follows: 
(1) Although the thrust force and the required torque of the driving motor increase the helical amplitude, wavelength, the wave number formed by the tails, the viscosity of environmental fluid, the rotating speed of the driving motor, the degrees of influence are various. When the helical amplitude is varied near some certain value, the microrobot will gain excellent dynamic properties.

(2) The wavelength formed by the tail, the radius of the tail, the amplitude of the tail and the wave number formed by the tails are the main factors to affect the thrust force. So if we need to adjust the swimming velocity of the micro microrobot, it only needs to change the values of above parameters.

(3) The wave number formed by the tail can affect the thrust force in some degree but not very dramatically. In this paper single objective optimization system consists of only one objective function prefers to increase the efficiency of propulsion microrobot. In order to have the maximum net forward velocity and minimize the required energy, the optimal values of the flagellum geometrical parameters for this microrobot are as follows

Optimal Geometrical Parameters

\begin{tabular}{cccc}
\hline $\begin{array}{c}\text { Wavelength } \\
(\mu \mathrm{m})\end{array}$ & $\begin{array}{c}\text { Amplitude } \\
(\mu \mathrm{m})\end{array}$ & $\begin{array}{c}\text { Radius } \\
(\mu \mathrm{m})\end{array}$ & $\begin{array}{c}\text { Wave Number } \\
(\mu \mathrm{m})\end{array}$ \\
\hline 0.708 & 0.115 & 0.0108 & 15 \\
\hline
\end{tabular}

According to the obtained results with applying different angular velocities as inputs, it is observed that microrobot designed not only has the ability of three dimensional movements but also the kinematic parameters of the such microrobot somehow optimized which can have applied in medicine and industrial applications with complex condition.

\section{Selected References}

1- Abbott, J.J., Nagy, Z., Beyeler, F. and Nelson, B.J., (2007), Robotics in the small, part I: Microbotics, Robotics \& Automation Magazine, IEEE, 14(2), p.92103. [DOI: 10.1109/MRA.2007.380641]

2- Abbott, J.J., Peyer, K.E., Lagomarsino, M.C., Zhang, L., Dong, L., Kaliakatsos, I.K. and Nelson, B.J., (2009), How should microrobots swim? The International Journal of Robotics Research, 28(11-12), p.1434-1447. [DOI: 10.1109/MRA.2007.380641]

3- Nelson, B.J., Kaliakatsos, I.K. and Abbott, J.J., (2010), Microrobots for minimally invasive medicine, Annual Review of Biomedical Engineering, 12(1), p.55-85. [DOI: 10.1146/annurev-bioeng-010510103409]

4- Purcell, E.M., (1977), Life at low Reynolds number, American Journal of Physics, 45(1), p.3-11. [DOI: 10.1119/1.10903]

5- Tottori, S., Zhang, L., Qiu, F., Krawczyk, K.K., Franco Obregon, A. and Nelson, B.J., (2012), Magnetic helical micro machines: Fabrication, controlled swimming and cargo transport, Advanced Materials, 24(6), p.811-816. [DOI: 10.1002/adma.201103818]

6- Zhang, L., Abbott, J.J., Dong, L., Kratochvil, B.E., Bell, D. and Nelson, B.J, (2009), Artificial bacterial flagella: Fabrication and magnetic control. Applied Physics Letters, 94(6), p. 064107-3 [DOI: 10.1063/1.3079655]

7- Fukuda, T., Kawamoto, A., Arai, F. and Matsuura, H., (1994), Mechanism and Swimming Experiment of Micro Mobile Robot in Water, Proc. of IEEE International Workshop on Micro Electro Mechanical Systems (MEMS'94), IEEE, New York, p. 273-278. [DOI: 10.1109/ROBOT.1994.351388]

8- Guo, S., Hasegaw, Y., Fukuda, T. and Asaka, K., (2001), Fish Like Underwater Microrobot with Multi $D O F$, Proceedings of 200 International Symposium on Micro mechatronics and Human Science, IEEE, Nahoya, Japan, p. 63-68. [DOI: 10.1109/MHS.2001.965223]

9- Jung, J., Kim, B., Tak, Y. and Park, J., (2003), Undulatory Tadpole Robot (Tad Rob) Using Ionic Polymer Metal Composite IMPC Actuator, Proceedings of 2003 IEEE International Conference on Intelligent Robots and Systems, IEEE, New York, p. 2133-2138. [DOI: 10.1109/IROS.2003.1249186]

10- Zhang, Y., Wang, Q., Zhang, P., Wang, X. and Mei, T., (2004), Dynamic Analysis and Experiment of a 3 $\mathrm{mm}$ Swimming Microrobot, Proceedings of 2004 IEEE International Conference on Intelligent Robots and Systems, IEEE, New York, p. 1746-1750. [DOI: 10.1109/IROS.2004.1389648]

11- Honda, T., Arai, K. and Ishiyama, K., (1999), Effect of Micro Machine Shape on Swimming Properties of the Spiral Type Magnetic Micro Machine, IEEE Trans. Magn., 35, p. 3688-3690. [DOI: 10.1109/20.800632] 12- Solovev, A. A., Mei, Y., Bermudez Urena, E., Huang, G. and Schmidt, O. G., (2009), Catalytic microtubular jet engines self propelled by accumulated gas bubbles, Small, 5 (14), p. 1688-92. [DOI: 10.1002/smll.200900021]

13- Hwang, G., Braive, R., Couraud, L., Cavanna, A., Abdelkarim, O., Robert Philip, I., Beveratos, A., Sagnes, I., Haliyo, S. and Regnier, S., (2011), Electroosmotic propulsion of helical nanobelt swimmers, The International Journal of Robotics Research, 30(7), p. 806-819. [DOI: 10.1002/smll.200900021]

14- Dreyfus, R., Baudry, J., Roper, M. L., Fermigier, M., Stone, H. A. and J. Bibette, (2005), Microscopic artificial swimmers, Nature, 437(7060), p. 862-865. [DOI: 10.1038/nature04090]

15- Yamazaki, A., Sendoh, M., Ishiyama, K., Ichi Arai, K., Kato, R., Nakano, M. and Fukunaga, H., (2004), Wireless micro swimming machine with magnetic thin film, Journal of Magnetism and Magnetic Materials, vol. 272, p. E1741-E1742. [DOI: 10.1016/j.jmmm.2003.12.337] 
16- Ghosh, A. and Fischer, P., (2009), Controlled propulsion of artificial magnetic nanostructured propellers, Nano Letters, 9(6), p. 2243-5. [DOI: $10.1021 / \mathrm{nl} 900186 \mathrm{w}]$

17- Tottori, S., Zhang, L., Qiu, F., Krawczyk, K. K., Franco Obregon, A. and Nelson, B. J., (2012), Magnetic helical micromachines: Fabrication, controlled swimming, and cargo transport, Advanced materials, 24(6), p. 811-816. [DOI: 10.1002/adma.201103818]

18- Zhang, L., Abbott, J. J., Dong, L., Peyer, K. E., Kratochvil, B. E., Zhang, H., Bergeles, C. and Nelson, B. J., (2009), Characterizing the swimming properties of artificial bacterial flagella, Nano Letters, 9(10), p. 3663-7. [DOI: 10.1021/n1901869j]

19- Kummer, M. P., Abbott, J. J., Kratochvil, B., Borer, R., Sengul, A. and Nelson, B. J., (2010), OctoMag: An electromagnetic system for 5 DOF wireless micromanipulation, IEEE Transactions on Robotics, 26(6), p. 1006-1017. [DOI: 10.1109/TRO.2010.2073030]

20- Martel, S., Felfoul, O., Mathieu, J.-B., Chanu, A., Tamaz, S., Mohammadi, M., Mankiewicz, M. and Tabatabaei, N., (2009), MRI based medical nanorobotics platform for the control of magnetic nanoparticles and flagellated bacteria for target interventions in human capillaries, The International Journal of Robotics Research, 28(9), p. 1169-1182. [DOI: $10.1177 / 0278364908104855]$

21- Hyung Kim, D., Seung Soo Kim, P., Agung Julius, A. and Jun Kim, M., (2012 ), Three dimensional control of Tetrahymena pyriformis using artificial magnetotaxis, Applied Physics Letters, 100(5), p. 053702. [DOI: 10.1063/1.3678340]

22- Kim, D. H., Liu, A., Diller, E. and Sitti, M., (2012), Chemotactic steering of bacteria propelled microbeads, Biomedical Micro devices, 14(6), p. 1009-1017. [DOI: 10.1007/s10544-012-9701-4]

23- Martel, S., and Mohammadi, M., (2010), Using a swarm of self propelled natural microrobots in the form of flagellated bacteria to perform complex microassembly tasks, in International Conference on Robotics and Automation, p. 500-505. [DOI: 10.1109/ROBOT.2010.5509752]

24- Behkam, B. and Sitti, M., (2006), Design methodology for biomimetic propulsion of miniature swimming robots, Journal of Dynamic Systems
Measurement and Control, Vol. 128, p. 36-43. [DOI: 10.1115/1.2171439]

25- Behkam, B. and Sitti, M., (2005), Modeling and testing of a biomimetic flagellar propulsion method for micro scale biomedical swimming robots. Proceedings of the IEEE/ASME International Conference on Advanced Intelligent Mechatronics, Monterey, USA, p. 37-42. [DOI: 10.1109/AIM.2005.1500962]

26- Brennen, C. and Winet, H., (1977), Fluid Mechanics of Propulsion by Cilia and Flagella. Annual Review of Fluid Mechanics, Vol. 9, p.339-398. [DOI: 10.1146/annurev.fl.09.010177.002011]

27- Gray, J. and Hancock, G., (1955), The propulsion of sea urchin spermatozoa, Journal of Experimental Biology, Vol. 32, p. 802-814.

28- Lighthill, J., (1976 ), Flagellar hydrodynamics, SIAM Review, vol. 18, p. 161-230. [DOI: 10.1137/1018040]

29- Chwang, T., and $\mathrm{Wu}, \mathrm{T} .$, (1971), A Note on the Helical Movement of Microorganisms, Proc. R. Soc. London, Ser. B, 178, p. 327-346. [DOI: 10.1098/rspb.1971.0068]

30- Kennedy, J. and Eberhart, R.C., (1995), Particle swarm optimization, in: Proceedings of the IEEE International Conference on Neural Networks IV, p. 1942-1948. [DOI: 10.1109/ICNN.1995.488968]

31- Eberhart, R.C., Dobbins, R. and Simpson, P.K., (1996), Computational intelligence PC tools, Morgan Kaufmann Publishers, Boston.

32- Engelbrecht, A.P., (2002), Computational Intelligence: An Introduction, John Wiley \& Sons, Chichester.

33- Engelbrecht, A.P., (2005), Fundamentals of Computational Swarm Intelligence, John Wiley \& Sons, Chichester.

34- Eberhart, R.C. and Kennedy, J., (1995), a new optimizer using particle swarm theory, in: Proceedings of the Sixth International Symposium on Micro Machine and Human Science, p. 39-43. [DOI: 10.1109/MHS.1995.494215]

35- Ratnaweera, A. and Halgamuge, S.K., (2004), Self organizing hierarchical particle swarm optimizer with time varying acceleration coefficient computation, IEEE Transactions on Evolutionary Computation 8 p. 240-255. [DOI: 10.1109/TEVC.2004.826071] 